\title{
Evaluation of Macroscopic Properties in the Direct Simulation Monte Carlo Method
}

\author{
Quanhua Sun* and Iain D. Boyd ${ }^{\dagger}$ \\ University of Michigan, Ann Arbor, Michigan 48109
}

\begin{abstract}
General expressions for evaluating macroscopic properties in the direct simulation Monte Carlo (DSMC) method are examined using numerical examples. DSMC simulations show that the evaluations (both statistically dependent and independent) generally follow Gaussian distributions except that the temperature evaluations follow chi-square distributions, whereas their statistical errors mainly depend on the sample size. To reduce the statistical errors for macroscopic properties required during DSMC simulations, a subrelaxation technique is proposed to build up the sample size by including the previous history using a relaxation factor. It is found that the subrelaxation technique helps reduce the statistical errors as if the sample size were increased by a factor of the reciprocal of the relaxation factor. This property makes use of the technique attractive for many DSMC applications.
\end{abstract}

\begin{tabular}{lll} 
& \multicolumn{1}{c}{ Nomenclature } \\
$E$ & $=$ & statistical error \\
$F_{N}$ & $=\begin{array}{l}\text { number of real molecules represented by one } \\
\text { simulated particle }\end{array}$ \\
$k$ & $=$ & Boltzmann constant \\
$m$ & $=$ & mass of molecules \\
$N$ & $=$ & number of particles in a cell or number of time steps \\
$n$ & $=$ & number density \\
$T$ & $=$ & temperature \\
$V$ & $=$ & velocity \\
$V o l$ & $=$ & volume of computational cell \\
$\Gamma$ & $=$ & gamma function \\
$\theta$ & $=$ & relaxation factor \\
$\rho$ & $=$ & correlation coefficient \\
$\sigma$ & $=$ & standard deviation of a distribution
\end{tabular}

Subscripts

$i=$ index of velocity component or of time step

$j \quad=$ index of time step

$t \quad=$ time step

$x, y, z=$ velocity component in $x, y$, or $z$ direction

$0=$ average

\section{Introduction}

$\mathbf{T}$ HE direct simulation Monte Carlo (DSMC) method $^{1}$ has been widely used for solving problems from many fields, including rarefied atmospheric gas dynamics, ${ }^{2}$ materials processing, ${ }^{3}$ microfluidics, ${ }^{4}$ and vacuum systems. ${ }^{5}$ Whereas the particle representation of gas flows has enabled the DSMC method to include flow physics at the molecular level, it also gives rise to statistical fluctuations when evaluating macroscopic properties. Two related questions are therefore how to evaluate macroscopic properties and how to reduce the statistical fluctuations.

Studies on the evaluation of macroscopic properties and their statistical fluctuations in DSMC simulations have been reported by

Received 29 July 2004; revision received 29 September 2004; accepted for publication 7 October 2004. Copyright (C) 2005 by the American Institute of Aeronautics and Astronautics, Inc. All rights reserved. Copies of this paper may be made for personal or internal use, on condition that the copier pay the $\$ 10.00$ per-copy fee to the Copyright Clearance Center, Inc., 222 Rosewood Drive, Danvers, MA 01923; include the code 0887-8722/05 $\$ 10.00$ in correspondence with the CCC.

*Postdoctoral Research Fellow, Department of Aerospace Engineering. Senior Member AIAA.

†Professor, Department of Aerospace Engineering. Associate Fellow AIAA. many investigators, including Bird, ${ }^{1}$ Garcia,${ }^{6}$ Tysanner and Garcia, ${ }^{7}$ Chen and Boyd,${ }^{8}$ and Hadjiconstantinou et al. ${ }^{9}$ Previous studies derived general expressions for the evaluations and their statistical fluctuations. However, distributions of evaluations have not been examined. One outcome is the confusion about the evaluation of temperature when the sample size is small. Furthermore, understanding general evaluations is especially important for many applications where the number of samples is very limited. One example is the instantaneous evaluation of the average number of simulated particles used in determining the collision rate during DSMC simulations. ${ }^{1}$ Another example is the temperature evaluation for simulations where temperature-dependent models are implemented. ${ }^{10}$ Additional examples include hybrid methods where macroscopic properties are required when the DSMC method is coupled with a continuum approach. ${ }^{11}$ All of these examples involve instantaneous evaluations of macroscopic properties. Inaccurate evaluations either introduce numerical error to simulation results or greatly increase the computational cost and thus motivate the development and application of techniques to reduce statistical fluctuations for DSMC simulations.

Endeavors have been made recently for the reduction of the statistical fluctuations in DSMC simulations. One of the most recent techniques is the information-preservation (IP) method, in which each simulated particle in a DSMC simulation preserves additional information at the macroscopic level that is used to evaluate the macroscopic properties. It has been shown that the IP method can greatly decrease the statistical fluctuations when simulating lowspeed flows, ${ }^{12}$ but further development is required to accurately capture strong nonequilibium behavior (e.g., shock structure) for highspeed flows. ${ }^{13}$ Another variant ${ }^{14}$ of the DSMC method is based on the relationship between the statistical fluctuation and the molecular mass, where the so-called molecular block model DSMC method can reduce the statistical fluctuation by increasing the molecular mass. However, this method also changes the flow Mach number for a simulation, and much work is needed to improve the method. ${ }^{15}$ Another technique reduces statistical fluctuations by applying a nonlinear filter to a DSMC solution as a postprocessor. ${ }^{16}$ However, filter techniques are seldom used in the DSMC method because filtering schemes generally take advantage of some known property of the object to be detected or analyzed.

In this paper, we examine general expressions and present a subrelaxation technique for evaluating macroscopic properties in the DSMC method. We first review theoretical expressions for evaluating the mean and statistical error of typical hydrodynamic properties. Then we explain the subrelaxation technique and derive related expressions. Next, we examine the general theoretical expressions and test the subrelaxation technique using three numerical examples. Finally, we close by discussing the need for precise evaluation of macroscopic properties. 


\section{Theoretical Expressions for Evaluation of Macroscopic Properties}

DSMC simulations are unsteady as they track the molecular behavior of simulated particles even for steady flows. They predict the macroscopic properties of a flow by sampling the particle information in each computational cell, and the particle samples can be accumulated by using either ensemble averaging for unsteady flows or time averaging for steady flows. Therefore, macroscopic properties are the statistical averages of appropriate particle properties, whereas their statistical fluctuations or statistical errors can be approximately derived based on statistical mechanics by assuming that samples are statistically independent. In this section, we review theoretical expressions for typical hydrodynamic properties and their statistical errors, and references are given where detailed derivations are omitted.

The average particle number $N_{0}$ in a cell is evaluated by averaging the number of particles $N$ in the cell over $N_{t}$ time steps. The mean and the statistical error $E$ of the particle number can be expressed as follows for dilute gases 9 :

$$
\begin{gathered}
N_{0}=\langle N\rangle \\
E_{N}=\sigma_{N} / \sqrt{N_{t}}=\sqrt{N / N_{t}}
\end{gathered}
$$

The statistical error is defined as the root mean square of the evaluation error, which is generally the standard deviation $\sigma$ of the single error distribution divided by the square root of the sample size as dictated by the central limit theorem. ${ }^{17}$ The number and mass density can be derived from the average particle number. For example, the number density $n$ can be evaluated as follows:

$$
n=N_{0} F_{N} / \mathrm{Vol}
$$

The flow velocity $V_{0}$ is evaluated as the average velocity of all sampled particles. The average \langle\rangle can be taken as the cumulative average [the average velocity of all $N\left(t_{j}\right)$ particles in a cell and over $N_{t}$ samples, Eq. (4)] or the sample average [the average mean velocity in a cell over $N_{t}$ samples, Eq. (5)].

$$
\begin{gathered}
\left\langle V_{i}\right\rangle_{\mathrm{CA}}=\frac{\sum_{j=1}^{N_{t}} \sum_{i=1}^{N\left(t_{j}\right)} V_{i}\left(t_{j}\right)}{\sum_{j=1}^{N_{t}} N\left(t_{j}\right)} \\
\left\langle V_{i}\right\rangle_{\mathrm{SA}}=\frac{1}{N_{t}} \sum_{j=1}^{N_{t}} \frac{1}{N\left(t_{j}\right)} \sum_{i=1}^{N\left(t_{j}\right)} V_{i}\left(t_{j}\right)
\end{gathered}
$$

However, Tysanner and $\mathrm{Garcia}^{7}$ recently pointed out that the sample average has a bias that arises from the correlation of momentum and density fluctuations in nonequilibrium fluids. The cumulative average is therefore used in the present investigation because it does not introduce bias. The expressions for evaluating velocity component $i$ can be derived as follows 9 :

$$
\begin{gathered}
V_{0, i}=\left\langle V_{i}\right\rangle \\
E_{V_{i}}=\sigma_{V_{i}} / \sqrt{N_{t} N_{0}}=\sqrt{k T_{0} / m N_{t} N_{0}}
\end{gathered}
$$

The evaluation of temperature is subtle because temperature is strictly defined only for equilibrium flows. The equilibrium temperature $T_{0}$ of a flow is evaluated based on the thermal fluctuations of individual particles. The following expressions show the mean and statistical error when the sample size is large enough, and only the translational modes are considered. ${ }^{9}$

$$
\begin{gathered}
T_{0}=(m / 3 k)\left(\left\langle V_{1}^{2}+V_{2}^{2}+V_{3}^{2}\right\rangle-\left\langle V_{1}\right\rangle^{2}-\left\langle V_{2}\right\rangle^{2}-\left\langle V_{3}\right\rangle^{2}\right) \\
E_{T}=\sigma_{T} / \sqrt{N_{t} N_{0}}=\sqrt{2 T_{0}^{2} / 3 N_{t} N_{0}}
\end{gathered}
$$

However, these expressions become invalid when the sample size is limited as noted by Roveda et al. ${ }^{11}$ The temperature is actually proportional to the variance of the velocity distribution and can be evaluated through the biased estimation of the variance of the velocity distribution, which can be expressed as follows:

$$
T_{0}=\frac{N_{t} N_{0}}{N_{t} N_{0}-1} \frac{m}{3 k}\left(\left\langle V_{1}^{2}+V_{2}^{2}+V_{3}^{2}\right\rangle-\left\langle V_{1}\right\rangle^{2}-\left\langle V_{2}\right\rangle^{2}-\left\langle V_{3}\right\rangle^{2}\right)
$$

This expression differs significantly from Eq. (8) when the sample size is small. The distribution of the temperature evaluation then follows a scaled chi-square distribution ${ }^{17}$ that can be derived as follows:

$$
\begin{aligned}
f\left(T_{0}\right) & =\frac{3 N_{t} N_{0}-1}{\left\langle T_{0}\right\rangle} \frac{1}{2^{\left(3 N_{t} N_{0}-1\right) / 2} \Gamma\left[\left(3 N_{t} N_{0}-1\right) / 2\right]} \\
& \times\left(\frac{\left(3 N_{t} N_{0}-1\right) T_{0}}{\left\langle T_{0}\right\rangle}\right)^{\left(3 N_{t} N_{0}-3\right) / 2} \exp \left(-\frac{\left(3 N_{t} N_{0}-1\right) T_{0}}{2\left\langle T_{0}\right\rangle}\right)
\end{aligned}
$$

This non-Gaussian distribution does not violate the central limit theorem because the temperature is not calculated as the simple average of a property. As expected for a chi-square distribution, the temperature distribution has positively skew and approaches the corresponding Gaussian distribution (with the same mean and variance) when the sample size increases. For simplicity, a Gaussian distribution is used as the theoretical expression for the temperature when the sample size $N_{t} N_{0}$ is larger than 33. The statistical error for the temperature evaluation is then calculated as follows:

$$
E_{T}=\sqrt{\frac{2 T_{0}^{2}}{3 N_{t} N_{0}-1}}
$$

\section{Subrelaxation Technique for Evaluating Macroscopic Properties}

For a typical DSMC simulation, roughly 20-100 particles are simulated in every computational cell. The statistical error is therefore very large for a sample size over one time step. For instance, if 50 particles are simulated in a cell for an argon flow having a flow velocity of $100 \mathrm{~m} / \mathrm{s}$ and a temperature of $300 \mathrm{~K}$, then the statistical error is about $14 \%\left(E_{N} / N_{0}\right)$ for the particle number, 35\% $\left(E_{V} / V_{0}\right)$ for the velocity, and $12 \%\left(E_{T} / T_{0}\right)$ for the temperature. To reduce the statistical error to an acceptable level, an averaging procedure can be used to increase the sample size. Such averaging can be spatial or temporal, with a temporal averaging preferred for steady simulations. A spatial average must assign weights to nearby cells, whereas a temporal average can use a subrelaxation technique to accumulate the sample size, which is discussed next.

Following the general relaxation technique, the temporal average $\overline{A_{j}}$ at time step $j$ is expressed as follows:

$$
\overline{A_{j}}=(1-\theta) \overline{A_{j-1}}+\theta A_{j}
$$

where $A_{j}$ is the instantaneous average based on the information in time step $j$. The relaxation technique is often called a subrelaxation technique when $\theta$ is less than one and an overrelaxation technique when $\theta$ is larger than one. If $\theta$ remains constant for a certain period, the following expressions can be derived:

$$
\begin{gathered}
\overline{A_{j}}=(1-\theta)^{j-i} \overline{A_{i}}+\theta \sum_{k=i+1}^{j}(1-\theta)^{j-k} A_{k} \\
\overline{A_{j}}=(1-\theta)^{j} A_{0}+\theta \sum_{k=1}^{j}(1-\theta)^{j-k} A_{k}
\end{gathered}
$$

where $A_{0}$ is given as the initial condition. From Eqs. (14) and (15), it is clear that the weight (coefficient) is not the same for all "history" 
$\left(A_{k}\right)$, and the history will be "forgotten" because of the subrelaxation (a small $\theta$ is used in present investigation), which is very important because earlier effects in the sampling process should be minimized. An alternative procedure is to average the samples using the same weight as follows:

$$
\overline{A_{j}}=\frac{1}{N_{t}} \sum_{k=1}^{N_{t}} A_{k}
$$

which means that an earlier sample has the same importance as the latest one. It will be shown later that this same-weight average is not preferred.
There is a tradeoff in the process of including previous samples: the temporal average always lags behind the status it should have. One natural thought to avoid the lag is to overrelax the temporal average after a period of subrelaxation. However, overrelaxation is unstable and requires additional knowledge as for filter schemes. A practical remedy is to exclude the previous lagged history and thus to prevent the history from introducing further error for later estimation. A correction to the average $\overline{A_{j}}$ can be derived by neglecting the terms prior to step $i$ as follows:

$$
{\overline{A_{j}}}^{\prime}=\overline{A_{j}}+\frac{(1-\theta)^{j-i}}{1-(1-\theta)^{j-i}}\left(\overline{A_{j}}-\overline{A_{i}}\right)
$$
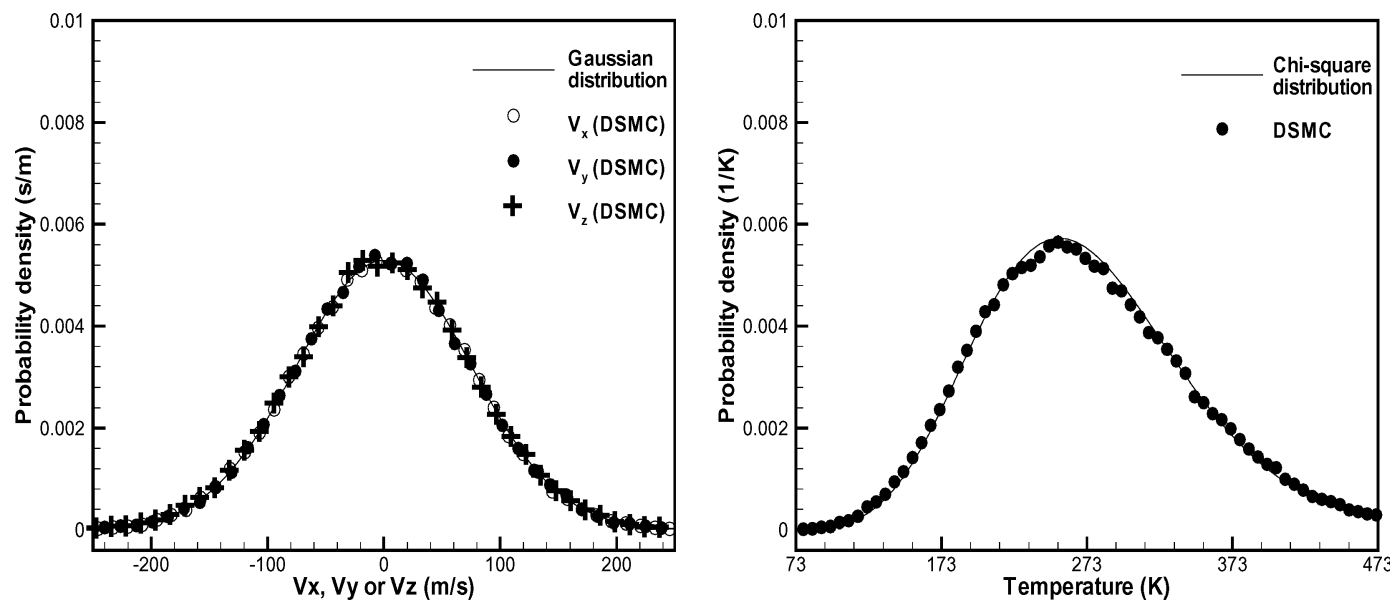

a) A sample size of 10 particles without using sub-relaxation technique
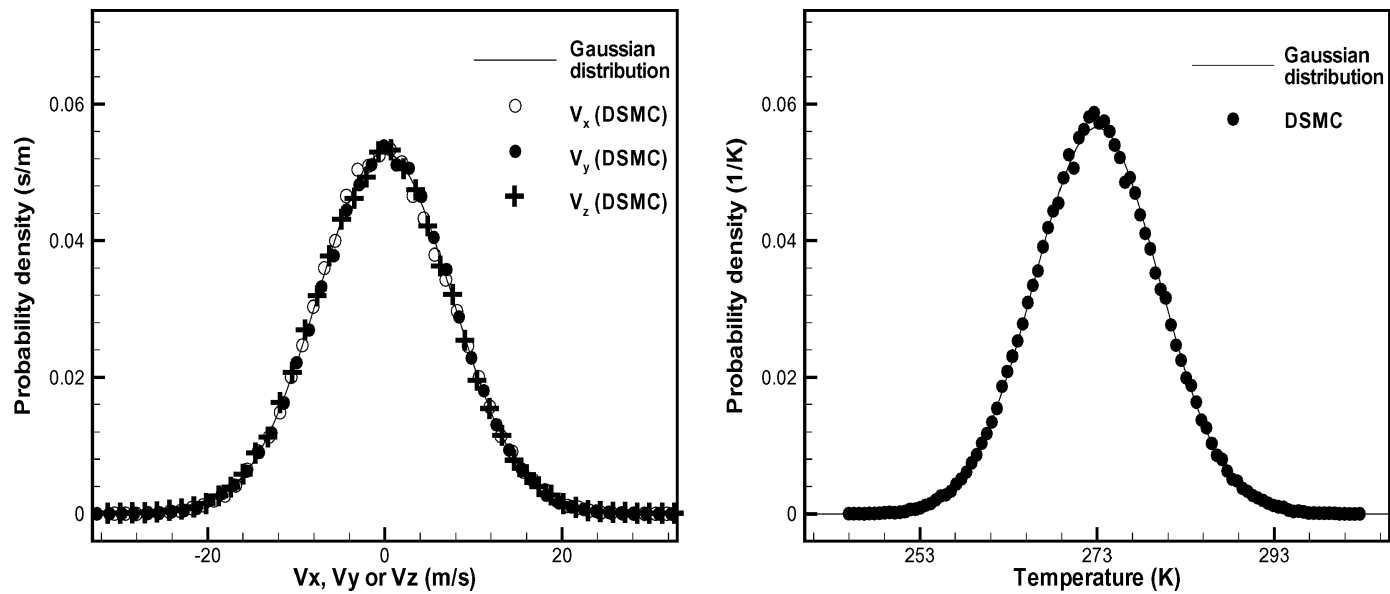

b) A sample size of 1,000 particles without using sub-relaxation technique
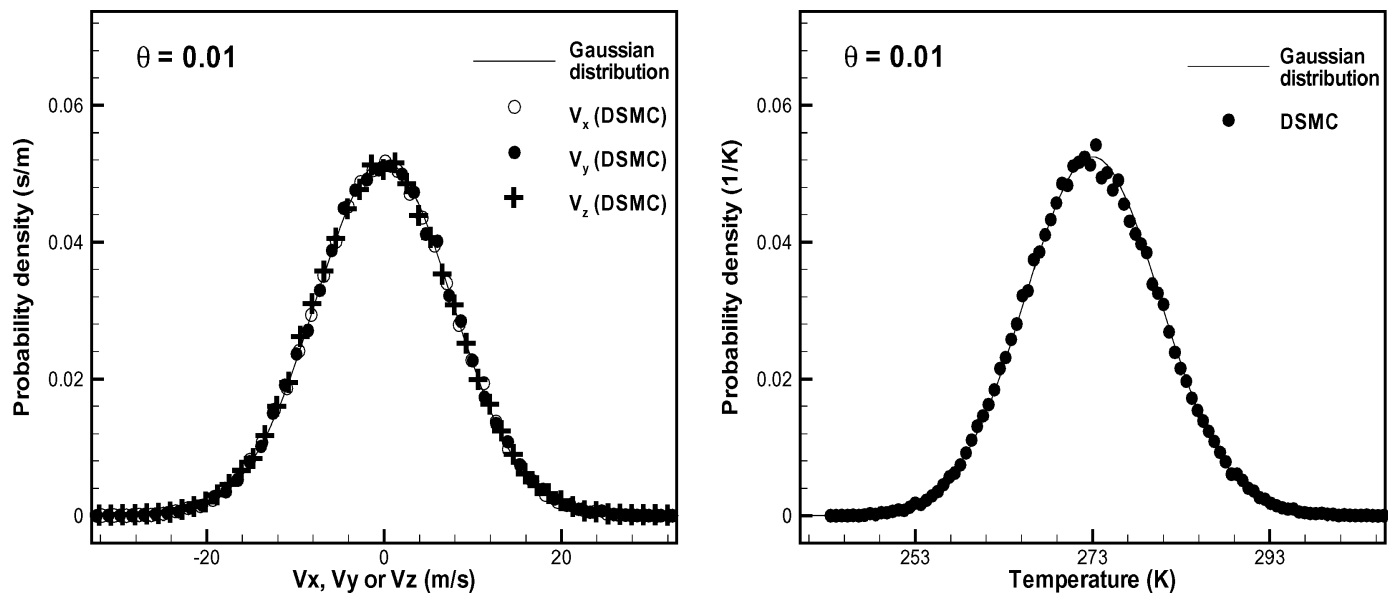

c) A sample size of 10 particles using sub-relaxation technique when $\theta=0.01$

Fig. 1 Probability density distributions of evaluated velocity and temperature for the box flow. 
A correction can only be made after the coefficient of the correction term is less than one in order to prevent the correction term from causing instability. Also to avoid saving all history of $\overline{A_{i}}$ for later corrections, a correction is only made when step $j$ meets a condition of $(j-i) \theta=1$ (other conditions can be used). Specifically, after a correction is made for step $i, \overline{A_{i}}$ is saved as $\overline{A_{i}}$, and the next step to have a correction is step $j=i+1 / \theta$. This condition of $(j-i) \theta=1$ is used for the results given in this paper unless otherwise indicated.

In summary, the proposed temporal average consists of three parts. First, an initial estimation (from initial conditions) is required [see Eq. (15)]. Second, a new average is evaluated based on the previous average and the instantaneous information using subrelaxation [Eq. (13)]. Third, a correction step can be used to prevent the earlier information from introducing further error [Eq. (17)]. The benefit of this temporal average is illustrated in the next section.

\section{Numerical Tests for Evaluating Macroscopic Properties and Their Statistical Errors}

The preceding expressions for evaluating macroscopic properties are examined using several numerical examples. The first example is a uniform box flow. The goals are to examine the expressions derived from statistics and to evaluate the temporal average where the subrelaxation technique is used. The second example is a flow in a two-dimensional driven cavity. The goals are similar to those of the first example except that the samples are statistically dependent and there are correlations among flow properties. The third example is a supersonic flow over a cylinder, which shows the effectiveness of the subrelaxation technique.

\section{Box Flow}

A uniform flow is simulated in a square box whose size is not important in this case. Simulated particles are generated based on the Maxwellian distribution having a temperature of $273 \mathrm{~K}$ and a zero mean velocity in every time step, so that statistical dependence between samples is avoided. The flow velocity and temperature of the argon gas are evaluated based on the particles in the box using Eqs. (6) and (10), respectively. The evaluations are performed 100,000 times with a fixed sample size in order to analyze the distributions of evaluated velocity and temperature. Figure 1 illustrates these distributions and the corresponding theoretical distributions.

Figure 1a shows the results when only 10 particles are simulated, and the temperature is calculated using $N_{0}=10$ and $N_{t}=1$. It is found that the evaluated flow velocity closely follows the Gaussian distribution using the standard deviation predicted by Eq. (7), and the temperature distribution follows the chi-square distribution as shown in Eq. (11). Obvious skewness is observed in the temperature distribution when the sample size is 10 , and the skewness disappears when the sample size is increased to 1000 particles $\left(N_{0}=1000\right.$ and $N_{t}=1$ ) as shown in Fig. 1b, where the chi-square distribution is replaced by the corresponding Gaussian distribution as explained earlier. Distributions of evaluations using the subrelaxation technique are plotted in Fig. 1c, where 10 particles are simulated and 0.01 is specified as the relaxation factor. The evaluations are obtained in every 100 time steps (recall that DSMC simulations are unsteady), which avoids the statistical dependence among evaluations. Specifically, the subrelaxation technique is applied to include effects of previous particle properties, but only the evaluations in every 100 time steps are analyzed to obtain the distribution. It is clear that the evaluated velocity and temperature closely follow the fitted Gaussian distributions, and they are close to the distributions (Fig. 1b) when 1000 particles are sampled.

To evaluate effects of the sample size and the relaxation factor, the statistical errors of the evaluations are plotted in Fig. 2. Clearly, the statistical errors can be decreased by either increasing the sample size or reducing the relaxation factor. The subrelaxation technique reduces the statistical errors by including the previous history as if the sample size were increased by a factor of the reciprocal of the relaxation factor, which provides a great improvement for the evaluation of the macroscopic properties. The reduction of the statistical error caused by the subrelaxation technique, however, does

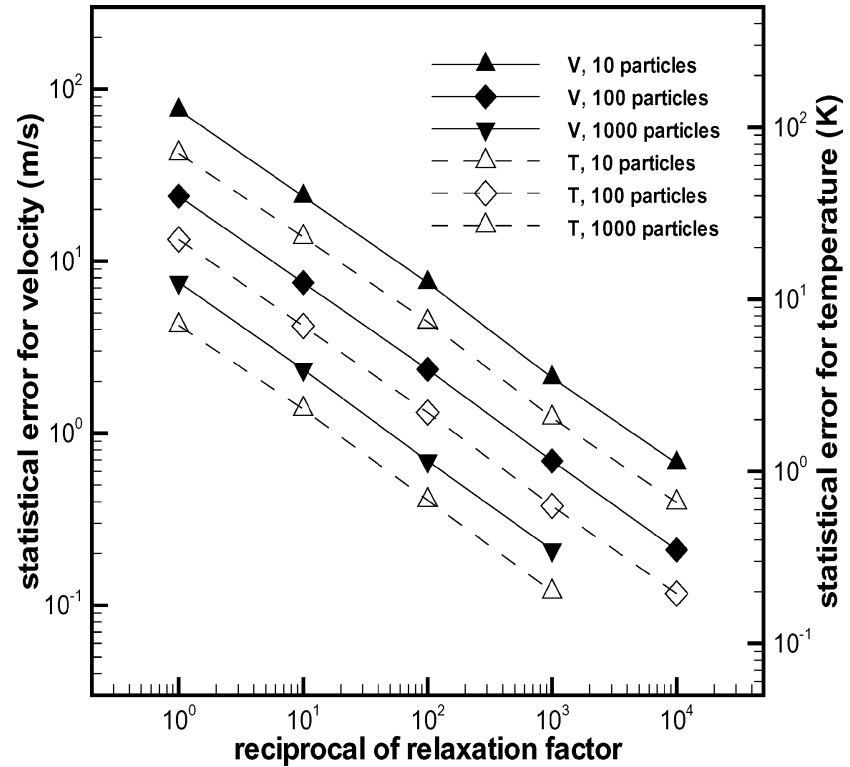

Fig. 2 Statistical errors predicted by DSMC simulations for the box flow.

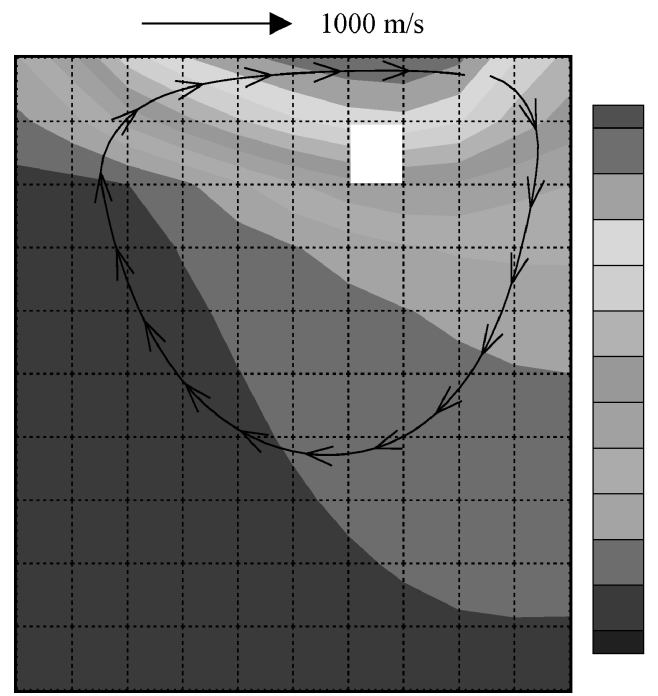

Fig. 3 Illustration of the two-dimensional driven cavity flow.

not violate the central limit theorem because the statistical error should decrease as the previous history is projected to the information at one time step. Also, there is roughly a $32 \%$ possibility for an evaluation to have an error larger than the defined statistical error.

\section{Two-Dimensional Driven Cavity Flow}

The driven cavity flow is used to further study the evaluation of macroscopic properties where samples are statistically dependent. A microcavity having $1 \mu$ in both length and height is driven by a plate having a velocity of $1000 \mathrm{~m} / \mathrm{s}$ and is divided into $10 \times 10$ cells for simulations. The temperature of both cavity wall and plate is kept at $273 \mathrm{~K}$. If the plate were at rest, the mean free path of the argon molecules is about one-third of the cavity length, and the time step is about one-third of the mean molecular collision time. The flow is sampled in every time step, and the results are illustrated in Fig. 3 where the contours indicate the temperature field. There are correlations among flow properties, and samples are statistically dependent. Therefore, evaluation of macroscopic properties for this flow can be different from that for the uniform box flow. As an example, analysis is given for the evaluated macroscopic data in the "white" cell as indicated in Fig. 3, where the data are obtained after the flow reaches a steady state. 

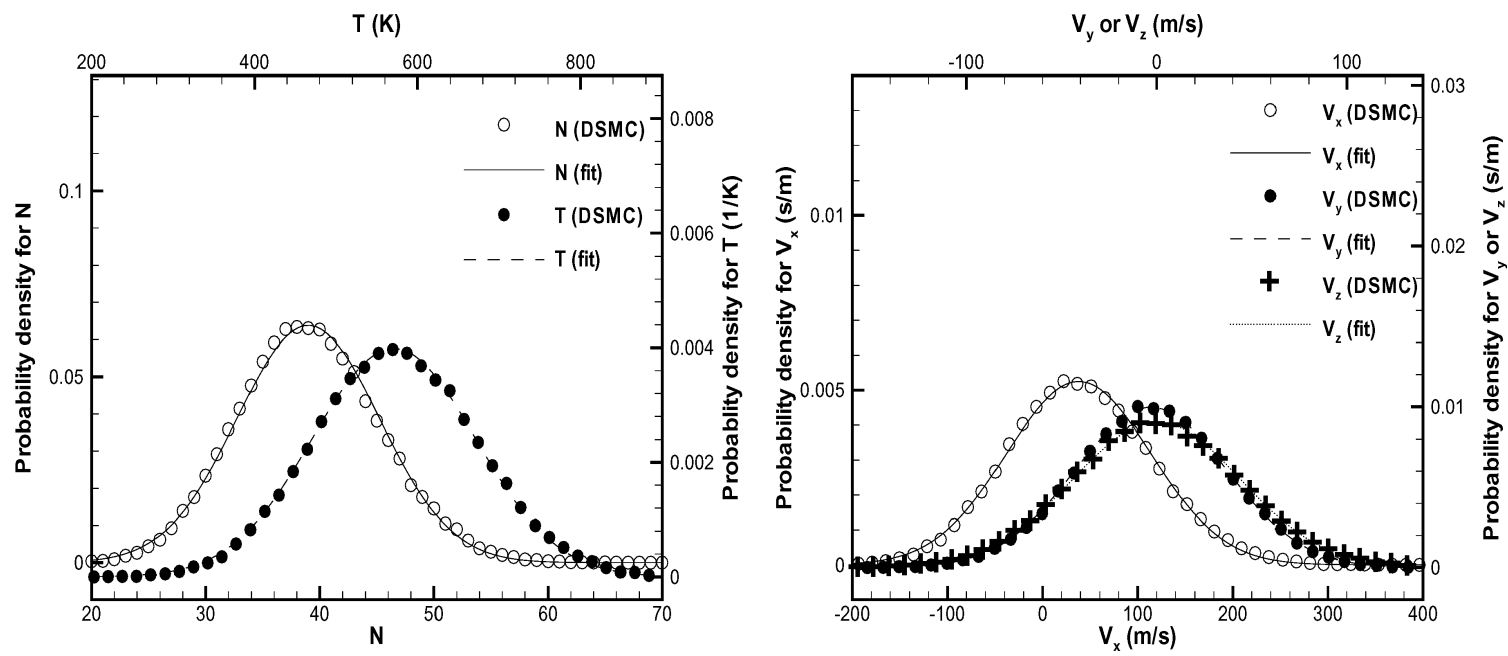

a) A sample size over 1 time step without using sub-relaxation technique
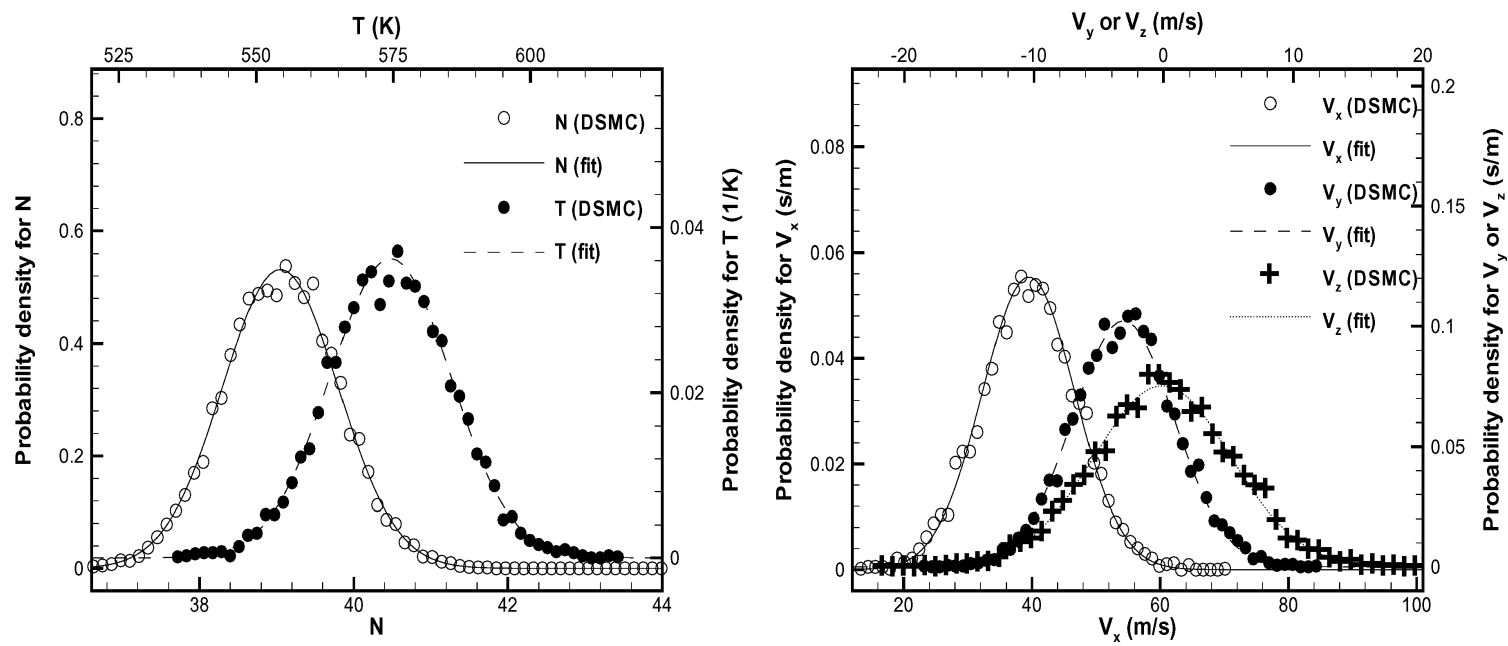

b) A sample size over 100 time steps without using sub-relaxation technique
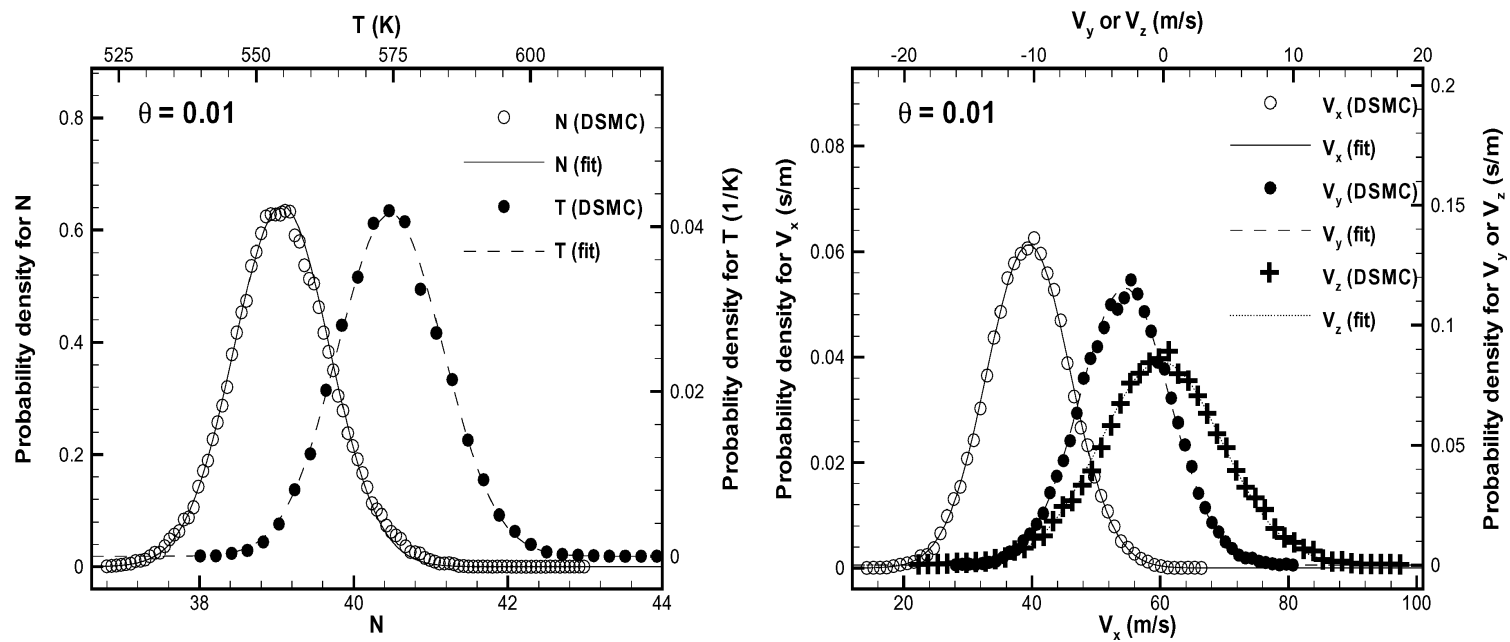

c) A sample size over 1 time step using sub-relaxation technique when $\theta=0.01$

Fig. 4 Probability density distributions of evaluated properties for the driven cavity flow.

The correlations among flow properties can be illustrated using the correlation coefficient, ${ }^{17}$ which is defined as follows:

$$
\rho(a, b)=\frac{\langle\delta a \delta b\rangle}{\sqrt{\left\langle\delta a^{2}\right\rangle\left\langle\delta b^{2}\right\rangle}}
$$

where $\delta a=a-\langle a\rangle$ and $\delta b=b-\langle b\rangle$. Using data obtained in the designated cell, obvious correlations are found for the following prop- erties: $\rho\left(V_{x}, V_{y}\right)=-0.40, \rho\left(V_{x}, T\right)=0.65$, and $\rho\left(V_{y}, T\right)=-0.51$. The sampled macroscopic properties in the designated cell are plotted in Fig. 4, which shows their distributions and fitted Gaussian distributions. The properties investigated include the simulated particle number, temperature, and velocities. The distributions are Gaussian although the samples are statistically dependent, and the statistical errors decrease when the sample size increases or the relaxation factor decreases. The scattered distributions in Fig. $4 \mathrm{~b}$ are as a result 


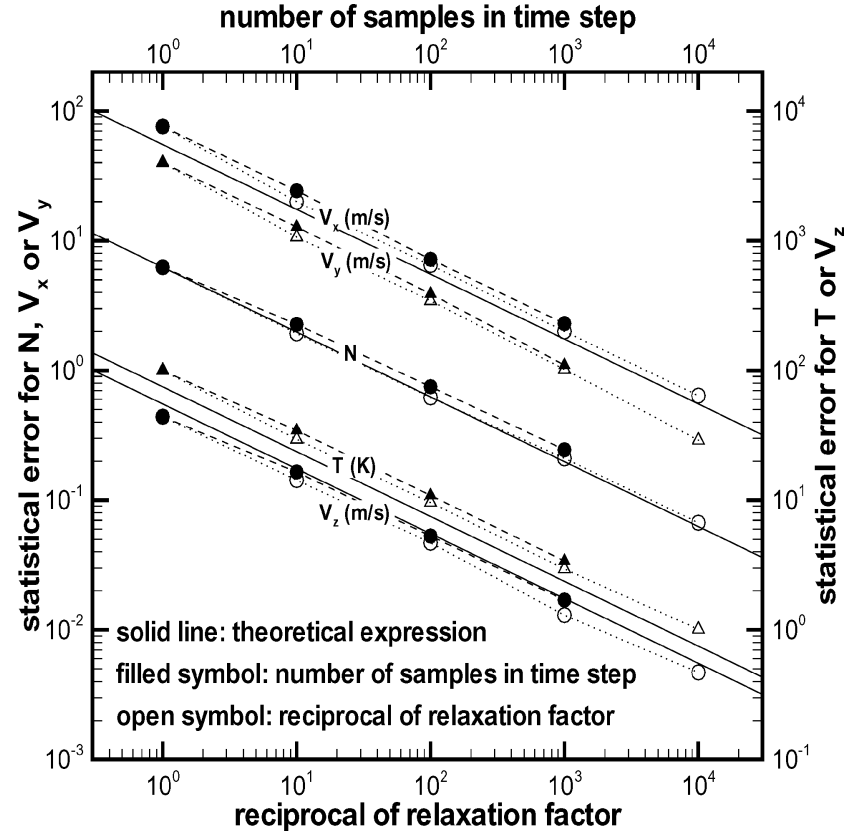

Fig. 5 Statistical errors predicted by DSMC simulations for the driven cavity flow.

of the small number of evaluations performed. The mean values of the evaluations are nearly the same for the same properties regardless of the sample size. The statistical errors for the evaluations are shown in Fig. 5. Again, a relaxation factor is able to decrease the statistical errors as if the sample size were increased by a factor of the reciprocal of the relaxation factor. It is found that the measured statistical errors do not always follow the expressions predicted by statistics where samples are assumed independent. In general, dependent samples exhibit larger statistical errors than independent samples when the sample size is the same. For instance, the statistical error for the simulated particle number drops slower than predicted, which means that more samples are needed to reduce the statistical error to the same desired level. The correlations among flow properties also make prediction of statistical errors difficult. For example, compared with theoretical values, the velocity component parallel to the plate $\left(V_{x}\right)$ displayed more statistical scatter, whereas the velocity component normal to the plate $\left(V_{y}\right)$ showed less statistical scatter. The correlation effects on the statistical scatters, however, need further investigation.

\section{Supersonic Flow over a Cylinder}

A supersonic flow over a cylinder is used to demonstrate the subrelaxation technique when evaluating macroscopic properties. The cylinder has a radius of $0.1 \mathrm{~m}$, and the wall is kept at $273 \mathrm{~K}$, the same temperature of the freestream. The freestream is argon having a Mach number of 5 and a Knudsen number of 0.05 based on the cylinder radius. The computational domain is shown in Fig. 6, where roughly 10,000 unstructured triangular cells are used.

Analysis of the data for the cell located at $(-0.15 \mathrm{~m}, 0.05 \mathrm{~m})$ is performed to show the progress of the simulation. It is found that statistical errors exceed flow properties if samples are limited to one time step (about 35 particles are simulated during the steady state). Hence, the relaxation factor should be very small to provide a meaningful estimation for a flow property. The same-weight average [Eq. (16)] is also implemented for the sake of comparison. Histories of the evaluated average number of particles are plotted in Fig. 7, where only data in every 10 time steps are shown. When the relaxation factor is 0.1 or 0.01 , the estimation follows the flow very closely, but the statistical error or fluctuation is large. On the other hand, when the relaxation factor is 0.001 , the estimation has small statistical error, but lags behind the flow significantly. The sudden jump in the plot is because of the correction step in the subrelaxation technique (see the subrelaxation technique section for details).

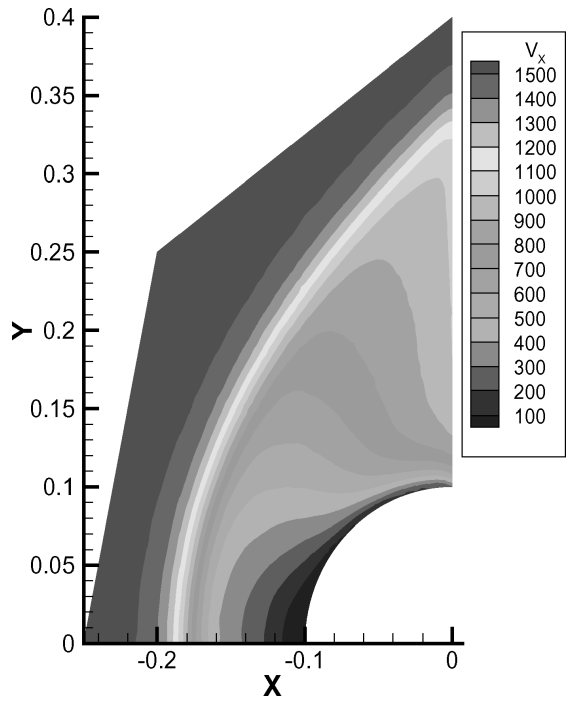

Fig. 6 Illustration of the supersonic flow over a cylinder.

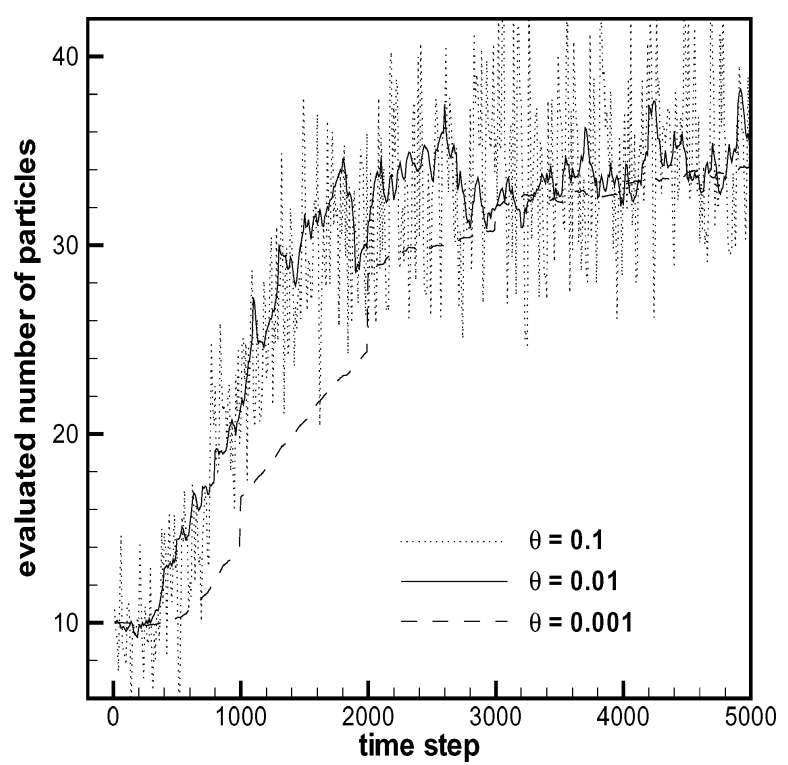

Fig. 7 History of evaluated average number of particles using the subrelaxation technique.

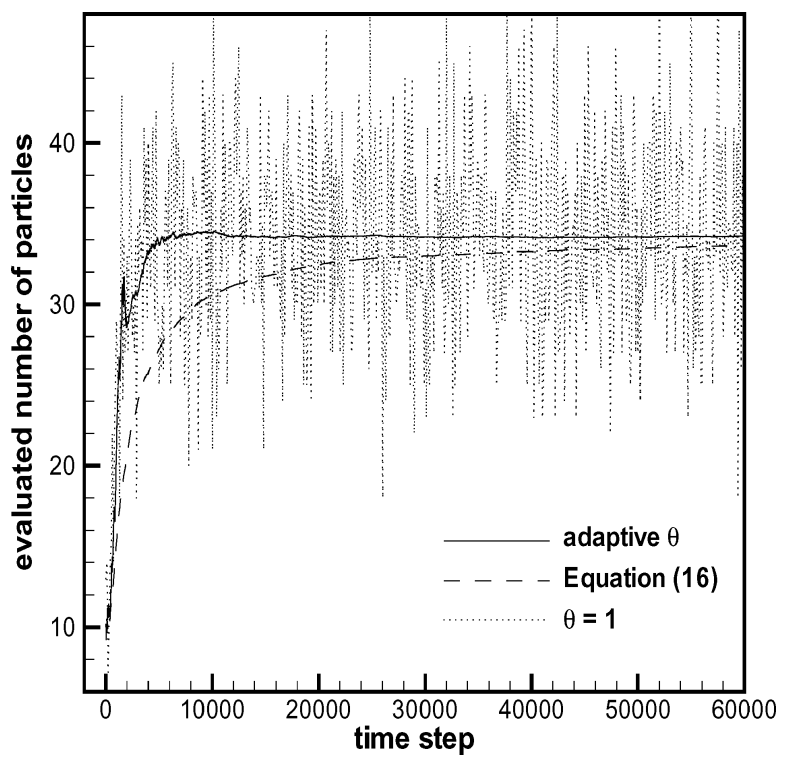

Fig. 8 Comparison of evaluated average numbers of particles using different temporal averages. 
Therefore, a better usage of the subrelaxation technique is to combine several values for the relaxation factor: a large relaxation factor should be used where the flow changes quickly and a small one is to be used after the flow reaches a steady state. Figure 8 illustrates this strategy by showing the history. The relaxation factor starts at 0.01 and is decreased to 0.001 after 2000 time steps. Then, during the steady simulation, it is adjusted to $1 /\left(t_{\text {step }}-4000\right)$ after 5,000 time steps, and the correction step is also neglected. The figure shows that the new evaluation follows the flow (as indicated by the case when $\theta=1$ ) very well, whereas the same-weight average always underestimates the flow. (This flaw of the same-weight average is often covered up by the statistical error.) Therefore, for the sake of accuracy and cost, the subrelaxation technique should be used in DSMC simulations that require macroscopic estimates based on limited sample sizes and/or transient phenomena.

\section{Conclusions}

The direct simulation Monte Carlo (DSMC) method is a particle approach where statistical errors always exist. This paper studied evaluations of typical hydrodynamic properties using numerical examples where both dependent and independent samples were considered. Although many numerical results agreed with theoretical expressions derived in the literature, care must be given to the temperature evaluation when the sample size is small and to the statistical errors when samples are statistically dependent. The temperature evaluation required a finite-sample correction, and the distribution of evaluations followed the chi-square distribution. When the samples were statistically dependent, the statistical errors did not always decrease according to the square root of the sample size.

This paper also proposed applying the subrelaxation technique in DSMC simulations for property evaluation. This was the first time that such an averaging scheme was discussed in detail for DSMC applications. The subrelaxation technique built up the sample size by including the previous history and was able to reduce statistical errors as if the sample size were increased by a factor of the reciprocal of the relaxation factor. Evaluations showed some time lag that is acceptable for steady simulations.

Subrelaxation is a simple technique that provides estimations for macroscopic properties required during DSMC simulations and should not be regarded as a variant of the DSMC method. Although the technique only offers time-lagged estimations, this technique is still very useful because many DSMC applications only involve steady simulations. For instance, evaluating the average number of simulated particles is often encountered in DSMC simulations for evaluation of collision rates. The inefficient same-weight average that has been widely used should be replaced with the subrelaxation technique because this technique gives flexible options to evaluate the average quickly and precisely. The subrelaxation technique can help implement physical boundary conditions other than the traditional supersonic (or vacuum) outflow conditions because obtaining smooth values for the hydrodynamic variables at such boundaries becomes possible by using a small relaxation factor. The reduced statistical errors obtained using this technique can also help develop efficient hybrid DSMC-continuum approaches. As many temperature- dependent models have been implemented in the DSMC method, precise evaluation of temperature is important because a small departure of the temperature can cause a very large error in rate constants. Therefore, the proposed subrelaxation technique can be useful to many DSMC applications.

\section{Acknowledgment}

The authors greatly appreciate support from the Air Force Office of Scientific Research through Grant F49620-03-1-0123.

\section{References}

${ }^{1}$ Bird, G. A., Molecular Gas Dynamics and the Direct Simulation of Gas Flows, Oxford Univ. Press, New York, 1994.

${ }^{2}$ Kannenberg, K. C., and Boyd, I. D., "Three-Dimensional Monte Carlo Simulations of Plume Impingement," Journal of Thermophysics and Heat Transfer, Vol. 13, No. 2, 1999, pp. 226-235.

${ }^{3}$ Chen, G., and Boyd, I. D., "Numerical Scale-up Study of Silicon Deposition Using 2D Slit Nozzle Sources," Materials Science in Semiconductor Processing, Vol. 1, No. 2, Sept. 1998, pp. 141-152.

${ }^{4}$ Sun, Q., Boyd, I. D., and Candler, G. V., "Numerical Simulation of Gas Flow over Micro-Scale Airfoils," Journal of Thermophysics and Heat Transfer, Vol. 16, No. 2, 2002, pp. 171-179.

${ }^{5}$ Penko, P. F., Boyd, I. D., Meissner, D. L., and DeWitt, K. J., "Measurement and Analysis of a Small Nozzle Plume in Vacuum," Journal of Propulsion and Power, Vol. 9, No. 4, 1993, pp. 646-648.

${ }^{6}$ Garcia, A. L., "Nonequilibrium Fluctuations Studied by a Rarefied-Gas Simulation," Physical Review A, Vol. 34, No. 2, 1986, pp. 1454-1457.

${ }^{7}$ Tysanner, M. W., and Garcia, A. L., "Measurement Bias of Fluid Velocity in Molecular Simulations," Journal of Computational Physics, Vol. 196, No. 1, May 2004, pp. 173-183.

${ }^{8}$ Chen, G., and Boyd, I. D., "Statistical Error Analysis for the Direct Simulation Monte Carlo Technique," Journal of Computation Physics, Vol. 126, No. 2, July 1996, pp. 434-448.

${ }^{9}$ Hadjiconstantinou, N. G., Garcia, A. L., Bazant, M. Z., and He, G., "Statistical Error in Particle Simulations of Hydrodynamic Phenomena," Journal of Computational Physics, Vol. 187, No. 1, May 2003, pp. 274-297.

${ }^{10}$ Boyd, I. D., "Analysis of Vibration-Dissociation-Recombination Processes Behind Strong Shock Waves of Nitrogen," Physics of Fluids A, Vol. 4, No. 1, 1992, pp. 178-185.

${ }^{11}$ Roveda, R., Goldstein, D. B., and Varghese, P. L., "Hybrid Euler/Particle Approach for Continuum/Rarefied Flows," Journal of Spacecraft and Rockets, Vol. 35, No. 3, 1998, pp. 258-265.

${ }^{12}$ Sun, Q., and Boyd, I. D., "A Direct Simulation Method for Subsonic, Micro-Scale Gas Flows," Journal of Computational Physics, Vol. 179, No. 2, July 2002, pp. 400-425.

${ }^{13}$ Wang, W.-L., and Boyd, I. D., "A New Energy Flux Model in the DSMCIP Method for Nonequilibrium Flows," AIAA Paper 2003-3774, June 2003.

${ }^{14}$ Pan, L. S., Ng, T. Y., Xu, D., and Lam, K. Y., "Molecular Block Model Direct Simulation Monte Carlo Method for Low Velocity Microgas Flows," Journal of Micromechanics and Microengineering, Vol. 11, No. 3, 2001, pp. 181-188.

${ }^{15}$ Wang, M., and Li, Z., "Failure Analysis of the Molecular Block Model for the Direct Simulation Monte Carlo Method," Physics of Fluids, Vol. 16, No. 6, 2004, pp. 2122-2125.

${ }^{16}$ Kaplan, C. R., and Oran, E., "Nonlinear Filtering for Low-Velocity Gaseous Microflows," AIAA Journal, Vol. 40, No. 1, 2002, pp. 82-90.

${ }^{17}$ Box, G. E. P., Hunter, W. G., and Hunter, J. S., Statistics for Experimenters, Wiley, New York, 1978. 